\title{
Existence of Positive Solutions of One-Dimensional Prescribed Mean Curvature Equation
}

\author{
Ruyun Ma and Lingfang Jiang \\ Department of Mathematics, Northwest Normal University, Lanzhou 730070, China \\ Correspondence should be addressed to Ruyun Ma; ruyun_ma@126.com
}

Received 23 October 2013; Accepted 28 February 2014; Published 13 April 2014

Academic Editor: Yuji Liu

Copyright (C) 2014 R. Ma and L. Jiang. This is an open access article distributed under the Creative Commons Attribution License, which permits unrestricted use, distribution, and reproduction in any medium, provided the original work is properly cited.

We consider the existence of positive solutions of one-dimensional prescribed mean curvature equation $-\left(u^{\prime} /{\sqrt{1+u^{\prime 2}}}^{\prime}=\lambda f(u)\right.$, $0<t<1, u(t)>0, t \in(0,1), u(0)=u(1)=0$ where $\lambda>0$ is a parameter, and $f:[0, \infty) \rightarrow[0, \infty)$ is continuous. Further, when $f$ satisfies $\max \left\{u^{p}, u^{q}\right\} \leq f(u) \leq u^{p}+u^{q}, 0<p \leq q<+\infty$, we obtain the exact number of positive solutions. The main results are based upon quadrature method.

\section{Introduction and Preliminaries}

In this paper, we are interested in the existence of positive solution of one-dimensional prescribed mean curvature equation

$$
\begin{gathered}
-\left(\frac{u^{\prime}}{\sqrt{1+u^{\prime 2}}}\right)^{\prime}=\lambda f(u), \quad 0<t<1, \\
u(t)>0, \quad t \in(0,1), \\
u(0)=u(1)=0,
\end{gathered}
$$

where $\lambda>0$ is a parameter and $f$ satisfies $\max \left\{u^{p}, u^{q}\right\} \leq$ $f(u) \leq u^{p}+u^{q}, 0<p \leq q<+\infty$.

The existence of positive solutions of such type of prescribed mean curvature equations both in one and in higher dimension has been discussed in the last decades by several authors (see Habets and Omari [1, 2], Li and Liu [3], Pan [4], Obersnel and Omari [5, 6], and others [7-12]) in connection with various configurations of nonlinearities.

Habets and Omari [1] considered the case that the nonlinearity $f(u)=u^{p}$; they proved that if $0<p<1$, then there exist $\lambda_{*}$ and $\lambda^{*}$ with $0<\lambda_{*}<\lambda^{*}$ such that (1) has exactly one positive solution for $\lambda \in\left(0, \lambda_{*}\right] \cup\left\{\lambda^{*}\right\}$, exactly two solutions for $\lambda \in\left(\lambda_{*}, \lambda^{*}\right)$, and no positive solution for $\lambda>\lambda^{*}$; if $p=1$, then there exists $\lambda_{*}$ with $0<\lambda_{*}<\pi^{2}$ such that (1) has exactly one positive solution for $\lambda \in\left(\lambda_{*}, \pi^{2}\right)$ and no positive solution for $\lambda \in\left(0, \lambda_{*}\right] \cup\left[\pi^{2},+\infty\right)$; if $p>1$, then there exist $\lambda_{*}>0$ and $\lambda^{*}>0$ such that (1) has no positive solution for $\lambda \in\left(0, \lambda_{*}\right]$ and exactly one positive solution for $\lambda>\lambda_{*}$.

$\mathrm{Li}$ and Liu [3] studied the case that the nonlinearity $f(u)=u^{p}+u^{q}, 0<p<q<+\infty$; they proved that if $1<p<q<+\infty$, then (1) has at most one solution for any $\lambda>0$; there exist $0<\lambda_{1}<\lambda_{2}<+\infty$ such that (1) has no positive solution for $0<\lambda<\lambda_{1}$ and exactly one positive solution for $\lambda>\lambda_{2}$; if $0<p<q<1$, then (1) has at most two solutions for any $\lambda>0$; there exists $0<\lambda_{1}<\lambda_{2}<+\infty$ such that (1) has exactly one positive solution for $0<\lambda<\lambda_{1}$ and no positive solution for $\lambda>\lambda_{2}$.

Inspired by $[1,3]$, we naturally consider (1) with nonlinearity $f$ satisfying

$$
\max \left\{u^{p}, u^{q}\right\} \leq f(u) \leq u^{p}+u^{q}, \quad 0<p \leq q<+\infty .
$$

Denote $F_{\lambda}(s)=\lambda \int_{0}^{s} f(t) d t, \alpha(\lambda)=F_{\lambda}^{-1}(1)$.

Consider the following assumptions:

(H1) $f:[0, \infty) \rightarrow[0, \infty)$ is continuous, and $f^{\prime}(s) \geq 0$, $s>0$

(H2) $\max \left\{u^{p}, u^{q}\right\} \leq f(u) \leq u^{p}+u^{q}$, for $0<p \leq q<\infty$, $u>0$; 
(H3) for fixed $\lambda>0, f(s)-s f^{\prime}(s) \leq 0$ for $s \in(0, \alpha(\lambda))$;

(H4) for fixed $\lambda>0, f(s)-3 s f^{\prime}(s) \geq 0, s \in(0, \alpha(\lambda))$.

We will prove the following.

Theorem 1. Assume that (H1)-(H3) hold, and, if $1<p \leq q<$ $\infty$, then the following conclusions hold:

(1) for any $\lambda>0$, (1) has at most one solution;

(2) there exists $0<\lambda_{1}<\lambda_{2}<+\infty$ such that (1) has no solution for $0<\lambda<\lambda_{1}$ and has exactly one solution for $\lambda>\lambda_{2}$.

Theorem 2. Assume that (H1), (H2), and (H4) hold, and, if $0<p \leq q<1$, then the following conclusions hold:

(1) for any $\lambda>0$, (1) has at most two solutions;

(2) there exists $0<\lambda_{1}<\lambda_{2}<+\infty$ such that (1) has exactly one solution for $0<\lambda<\lambda_{1}$ and has no solution for $\lambda>\lambda_{2}$.

Remark 3. If $f(u)=\max \left\{u^{p}, u^{q}\right\}$ and $p=q$, then (2) reduces to $f(u)=u^{p}$, which has been considered by Habets and Omari [1]; $f(u)=u^{p}+u^{q}$ for $0<p<q<1$ and $1<p<q<+\infty$, which has been studied by Li and Liu [3], and the case $0<p<1<q<+\infty$ has been considered by Zhang and Feng [7].

Remark 4. The problem concerning exact number of solutions of semilinear equation

$$
\begin{gathered}
-u^{\prime \prime}=\lambda f(u), \quad u>0, t \in(0,1), \\
u(0)=u(1)=0,
\end{gathered}
$$

has been discussed by several authors with $f(u)=u^{p}+u^{q}$ and the related problems (see [13-16]).

\section{Quadrature Technique}

Let $u(t)$ be a solution of problem (1); then it is well known that $u(t)$ takes its maximum at $c=1 / 2, u(t)$ is symmetric with respect to $c, u^{\prime}(t)>0$ for $0 \leq t<c$, and $u^{\prime}(t)<0$ for $c<t \leq 1$. Hence, problem (1) is equivalent to the following problem defined on $[0, c]$ :

$$
\begin{gathered}
-\left(\frac{u^{\prime}}{\sqrt{1+u^{\prime 2}}}\right)^{\prime}=\lambda f(u), \\
u(t)>0, \quad t \in(0, c), \\
u(0)=u^{\prime}(c)=0 .
\end{gathered}
$$

Let $v=u^{\prime} / \sqrt{1+u^{\prime 2}}$. If $u(t)$ is a solution of (4) with $\rho=u(c)$, then $(u, v)$ is a solution of the following problem defined on $[0, c]$ :

$$
\begin{gathered}
u^{\prime}=\frac{v}{\sqrt{1-v^{2}}}, \quad v^{\prime}=-\lambda f(u), \\
u(0)=0, u(c)=\rho, v(c)=0 .
\end{gathered}
$$

From $H(t)=\sqrt{1-v^{2}(t)}-1-F_{\lambda}(u)$ satisfying

$$
\frac{d H(t)}{d t}=\frac{-v(t)}{\sqrt{1-v^{2}(t)}}\left(v^{\prime}(t)+\lambda f(u)\right)=0
$$

and $H(c)=-F_{\lambda}(\rho)$, we see that

$$
F_{\lambda}(\rho)-F_{\lambda}(u(t))=1-\frac{1}{\sqrt{1+u^{\prime 2}(t)}}
$$

Therefore,

$$
u^{\prime}=\frac{\sqrt{\left(F_{\lambda}(\rho)-F_{\lambda}(u)\right)\left[2-\left(F_{\lambda}(\rho)-F_{\lambda}(u)\right)\right]}}{1-\left(F_{\lambda}(\rho)-F_{\lambda}(u)\right)} .
$$

Then

$$
\frac{1-\left(F_{\lambda}(\rho)-F_{\lambda}(u)\right)}{\sqrt{\left(F_{\lambda}(\rho)-F_{\lambda}(u)\right)\left[2-\left(F_{\lambda}(\rho)-F_{\lambda}(u)\right)\right]}} d u=d t .
$$

Integrating (9) from 0 to $c$ leads to

$$
\int_{0}^{\rho} \frac{1-\left(F_{\lambda}(\rho)-F_{\lambda}(u)\right)}{\sqrt{\left(F_{\lambda}(\rho)-F_{\lambda}(u)\right)\left[2-\left(F_{\lambda}(\rho)-F_{\lambda}(u)\right)\right]}} d u=\frac{1}{2} .
$$

By substituting $t=0$ in (7), we get that $F_{\lambda}(\rho)<1$ and $0<\rho<$ $\alpha(\lambda)=F_{\lambda}^{-1}(1)$.

Let $T$ be defined on $\Sigma=\{(\lambda, \rho): \lambda \in(0, \infty), \rho \in$ $(0, \alpha(\lambda)]\}$ by

$$
T(\lambda, \rho)=\int_{0}^{\rho} \frac{1-\left(F_{\lambda}(\rho)-F_{\lambda}(u)\right)}{\sqrt{\left(F_{\lambda}(\rho)-F_{\lambda}(u)\right)\left[2-\left(F_{\lambda}(\rho)-F_{\lambda}(u)\right)\right]}} d u .
$$

For simplicity, we denote

$$
\begin{gathered}
\xi=\xi_{\lambda}(\rho, t)=F_{\lambda}(\rho)-F_{\lambda}(\rho t), \\
\xi^{\prime}=\frac{\partial}{\partial \rho} \xi_{\lambda}(\rho, t), \quad \xi^{\prime \prime}=\frac{\partial^{2}}{\partial \rho^{2}} \xi_{\lambda}(\rho, t), \\
\Delta F=F_{\lambda}(\rho)-F_{\lambda}(u), \quad \Delta \bar{f}=\lambda \rho f(\rho)-\lambda u f(u), \\
\Delta \bar{f}^{\prime}=\lambda \rho^{2} \frac{\partial}{\partial \rho} f(\rho)-\lambda u^{2} \frac{\partial}{\partial u} f(u) .
\end{gathered}
$$


It follows that

$$
T(\lambda, \rho)=\rho \int_{0}^{1} \frac{1-\xi}{\sqrt{\xi(2-\xi)}} d t .
$$

Lemma 5. $T(\lambda, \rho)$ has continuous derivatives up to the second order on $\Sigma$ with respect to $\rho$ and

$$
\begin{aligned}
& T_{\rho}^{\prime}(\lambda, \rho)= \int_{0}^{1} \frac{\xi(1-\xi)(2-\xi)-\rho \xi^{\prime}}{(\xi(2-\xi))^{3 / 2}} d t \\
&= \frac{1}{\rho} \int_{0}^{\rho} \frac{\Delta F(1-\Delta F)(2-\Delta F)-\Delta \bar{f}}{[\Delta F(2-\Delta F)]^{3 / 2}} d u, \\
& T_{\rho \rho}^{\prime \prime}(\lambda, \rho)= \frac{3}{\rho} \int_{0}^{1} \frac{(1-\xi)\left(\rho \xi^{\prime}\right)^{2}}{(\xi(2-\xi))^{5 / 2}} d t \\
&-\int_{0}^{1} \frac{2 \xi^{\prime}+\rho \xi^{\prime \prime}}{(\xi(2-\xi))^{3 / 2}} d t \\
&= \frac{1}{\rho^{2}} \int_{0}^{\rho}\left(\left(3(1-\Delta F) \Delta \bar{f}^{2}\right.\right. \\
&\left.\quad-\left(\Delta \bar{f}^{\prime}+2 \Delta \bar{f}\right) \Delta F(2-\Delta F)\right) \\
&\left.\times[\Delta F(2-\Delta F)]^{-5 / 2}\right) d u .
\end{aligned}
$$

Proof. From Habets and Omari [1], it is easy to obtain the results.

Lemma 6. Let (H1) and (H2) hold; $\alpha(\lambda)$ is strictly decreasing on $(0, \infty)$ with respect to $\lambda$, and

$$
\lim _{\lambda \rightarrow 0} \alpha(\lambda)=+\infty, \quad \lim _{\lambda \rightarrow+\infty} \alpha(\lambda)=0 .
$$

Proof. Since

$$
\begin{gathered}
\lambda \frac{1}{p+1} t^{p+1} \leq F_{\lambda}(t) \leq \lambda\left(\frac{1}{p+1}+\frac{1}{q+1}\right) t^{p+1}, \quad t<1, \\
\lambda \frac{t^{q+1}}{q+1} \leq F_{\lambda}(t) \leq \lambda\left(\frac{1}{p+1}+\frac{1}{q+1}\right) t^{q+1}, \quad t>1,
\end{gathered}
$$

we have

$$
\begin{aligned}
& \left(\frac{\lambda}{p+1}+\frac{\lambda}{q+1}\right)^{-1 /(p+1)} t^{1 /(p+1)} \\
& \leq F_{\lambda}^{-1}(t) \leq\left(\frac{\lambda}{p+1}\right)^{-1 /(p+1)} t^{1 /(p+1)}, \\
& t<\frac{\lambda}{p+1}+\frac{\lambda}{q+1}, \\
& \left(\frac{\lambda}{p+1}+\frac{\lambda}{q+1}\right)^{-1 /(q+1)} t^{1 /(q+1)} \\
& \leq F_{\lambda}^{-1}(t) \leq\left(\frac{\lambda}{q+1}\right)^{-1 /(p+1)} t^{1 /(p+1)}, \quad t>\frac{\lambda}{q+1} .
\end{aligned}
$$

Then

$$
\begin{aligned}
&\left(\frac{\lambda}{p+1}+\frac{\lambda}{q+1}\right)^{-1 /(p+1)} \leq F_{\lambda}^{-1}(1) \leq\left(\frac{\lambda}{p+1}\right)^{-1 /(p+1)}, \\
& 1<\frac{\lambda}{p+1}+\frac{\lambda}{p+1}, \\
&\left(\frac{\lambda}{p+1}+\frac{\lambda}{q+1}\right)^{-1 /(q+1)} \leq F_{\lambda}^{-1}(1) \leq\left(\frac{\lambda}{q+1}\right)^{-1 /(p+1)}, \\
& 1>\frac{\lambda}{q+1} .
\end{aligned}
$$

It follows that $\lim _{\lambda \rightarrow 0} \alpha(\lambda)=\infty, \lim _{\lambda \rightarrow+\infty} \alpha(\lambda)=0$.

From the fact that $F_{\lambda}(\alpha(\lambda))=1$, we have $\lambda \int_{0}^{\alpha(\lambda)} f(s) d s=$ 1 , and $\int_{0}^{\alpha(\lambda)} f(s) d s+\lambda f(\alpha(\lambda)) \alpha^{\prime}=0$; it follows from the assumption $(\mathrm{Hl})$ that

$$
\alpha^{\prime}=-\frac{\int_{0}^{\alpha} f(s) d s}{\lambda f(\alpha)}<0 .
$$

Then $\alpha(\lambda)$ is strictly decreasing on $(0, \infty)$.

Lemma 7. Let (H1)-(H3) hold, if $1<p \leq q<+\infty$ and $\lambda>0$. Then $T(\lambda, \rho)$ has the following properties:

(i) $\lim _{\rho \rightarrow 0} T(\lambda, \rho)=+\infty$;

(ii) $T(\lambda, \alpha(\lambda))>0$;

(iii) $T_{\rho}^{\prime}(\lambda, \rho)=(\partial / \partial \rho) T(\lambda, \rho)<0$, for $\rho \in(0, \alpha(\lambda)]$.

Proof. (i) From (H2),

$$
\begin{aligned}
F_{\lambda}(\rho)-F_{\lambda}(\rho t)= & \lambda \int_{\rho t}^{\rho} f(s) d s \\
\leq & \rho^{p+1} \frac{\lambda}{p+1}\left(1-t^{p+1}\right) \\
& +\rho^{q+1} \frac{\lambda}{q+1}\left(1-t^{q+1}\right) ;
\end{aligned}
$$


therefore,

$$
\begin{array}{r}
T(\lambda, \rho)=\rho \int_{0}^{1} \frac{1-\xi}{\sqrt{\xi(2-\xi)}} d t \\
\geq \rho \int_{0}^{1}\left(( 1 - \xi ) \left(\left[\rho^{p+1} \frac{\lambda}{p+1}\left(1-t^{p+1}\right)+\rho^{q+1}\right.\right.\right. \\
\left.\quad \times \frac{\lambda}{q+1}\left(1-t^{q+1}\right)\right] \\
\left.\quad \times(2-\xi))^{-1 / 2}\right) d t \\
=\rho^{(1-p) / 2 \int_{0}^{1}\left(( 1 - \xi ) \left(\left[\frac{\lambda}{p+1}\left(1-t^{p+1}\right)+\rho^{q-p}\right.\right.\right.} \\
\left.\quad \times \frac{\lambda}{q+1}\left(1-t^{q+1}\right)\right] \\
\left.\quad \times(2-\xi))^{-1 / 2}\right) d t .
\end{array}
$$

Hence, from $p>1$, then $\lim _{\rho \rightarrow 0} T(\lambda, \rho)=+\infty$.

(ii) Since $F_{\lambda}(\alpha(\lambda) t)>0$, for $t \in(0,1)$, thus

$$
T(\lambda, \alpha(\lambda))=\alpha(\lambda) \int_{0}^{1} \frac{F_{\lambda}(\alpha(\lambda) t)}{\sqrt{\xi(2-\xi)}} d t>0 .
$$

(iii) From Lemma 5, we know that $T_{\rho}^{\prime}(\lambda, \rho)=\int_{0}^{1}((\xi(1-$ $\left.\left.\xi)(2-\xi)-\rho \xi^{\prime}\right) /(\xi(2-\xi))^{3 / 2}\right) d t$, so

$$
\begin{aligned}
& \xi(1-\xi)(2-\xi)-\rho \xi^{\prime} \\
& \leq 2 \xi-\rho \xi^{\prime} \\
&= 2 F_{\lambda}(\rho)-\rho \lambda f(\rho) \\
&-\left[2 F_{\lambda}(\rho t)-\lambda \rho t f(\rho t)\right] \\
&= H(\lambda, \rho)-H(\lambda, \rho t), \\
& t \in(0,1), \quad \rho \in(0, \alpha(\lambda)],
\end{aligned}
$$

where $H(\lambda, s)=2 F_{\lambda}(s)-\lambda s f(s), s \in(0, \alpha(\lambda)]$.

From the assumption $(\mathrm{H} 3)$, we have that $(\partial / \partial s) H(\lambda, s)=$ $\lambda f(s)-\lambda s f^{\prime}(s)<0, s \in(0, \alpha(\lambda)]$; thus $\xi(1-\xi)(2-\xi)-\rho \xi^{\prime} \leq$ $2 \xi-\rho \xi^{\prime}<0$.

Lemma 8. Assume that (H1) and (H2) hold, and let $\eta(\lambda)=$ $T(\lambda, \alpha(\lambda))$. Then $\eta(\lambda)$ has the following properties:

(i) for $0<p \leq q<+\infty, \eta(\lambda)$ is continuous in $(0,+\infty)$;

(ii) for $0<p \leq q<+\infty, \lim _{\lambda \rightarrow 0} \eta(\lambda)=+\infty$, $\lim _{\lambda \rightarrow \infty} \eta(\lambda)=0$.
Proof. (i) Applying $\eta(\lambda)=T(\lambda, \alpha(\lambda))=\alpha(\lambda) \int_{0}^{1}\left(F_{\lambda}(\alpha t) /\right.$ $\left.\sqrt{1-F_{\lambda}^{2}(\alpha t)}\right) d t=\int_{0}^{\alpha(\lambda)}\left(F_{\lambda}(s) / \sqrt{1-F_{\lambda}^{2}(s)}\right) d s$, we may prove the following (26), (29) and (30) hold. For simplicity, we denote $\alpha(\lambda)$ by $\alpha$.

If $\alpha \leq 1$, then $\lambda s^{p+1} /(p+1) \leq F_{\lambda}(s) \leq \lambda s^{p+1} /(p+1)+$ $\lambda s^{q+1} /(q+1), s \in(0, \alpha)$, and from $F_{\lambda}(\alpha(\lambda))=1$, we get

$$
\begin{aligned}
1-F_{\lambda}^{2}(s)= & F_{\lambda}^{2}(\alpha(\lambda))-F_{\lambda}^{2}(s) \\
= & \left(F_{\lambda}(\alpha(\lambda))-F_{\lambda}(s)\right) \\
& \times\left(F_{\lambda}(\alpha(\lambda))+F_{\lambda}(s)\right) \\
\geq & \lambda^{2}\left(\frac{\alpha^{p+1}}{p+1}-\frac{s^{p+1}}{p+1}\right) \\
& \times\left(\frac{\alpha^{p+1}}{p+1}+\frac{s^{p+1}}{p+1}\right) \\
= & \lambda^{2}\left(\frac{\alpha^{2 p+2}}{(p+1)^{2}}-\frac{s^{2 p+2}}{(p+1)^{2}}\right),
\end{aligned}
$$

$$
s \in(0, \alpha) .
$$

So

$$
\begin{aligned}
& \alpha \int_{0}^{1} \frac{F_{\lambda}(\alpha t)}{\sqrt{1-F_{\lambda}^{2}(\alpha t)}} d t \\
& \quad=\int_{0}^{\alpha} \frac{F_{\lambda}(s)}{\sqrt{1-F_{\lambda}^{2}(s)}} d s \\
& \quad \leq \int_{0}^{\alpha} \frac{\left(\lambda s^{p+1} /(p+1)\right)+\left(\lambda s^{q+1} /(q+1)\right)}{\sqrt{\lambda^{2}\left(\left(\alpha^{2 p+2} /(p+1)^{2}\right)-\left(s^{2 p+2} /(p+1)^{2}\right)\right)}} d s \\
& \quad=\alpha \int_{0}^{1} \frac{\left(\lambda(\alpha t)^{p+1} /(p+1)\right)+\left(\lambda(\alpha t)^{q+1} /(q+1)\right)}{\sqrt{\lambda^{2}\left(\left(\alpha^{2 p+2} /(p+1)^{2}\right)-\left((\alpha t)^{2 p+2} /(p+1)^{2}\right)\right)}} d t \\
& \quad \leq \alpha \int_{0}^{1} \frac{t^{p+1}+\alpha^{q-p} t^{q+1}}{\sqrt{1-t^{2 p+2}}} d t .
\end{aligned}
$$

Since $\alpha \leq 1$, we have from (25) that

$$
\alpha \int_{0}^{1} \frac{t^{p+1}+\alpha^{q-p} t^{q+1}}{\sqrt{1-t^{2 p+2}}} d t \leq 2 \int_{0}^{1} \frac{t^{p+1}}{\sqrt{1-t^{2 p+2}}} d t
$$

If $\alpha>1$, then $\lambda s^{p+1} /(p+1) \leq F_{\lambda}(s) \leq\left(\lambda s^{p+1} /(p+1)\right)+$ $\left(\lambda s^{q+1} /(q+1)\right), s \in(0,1)$ and $\left(\lambda s^{q+1} /(q+1)\right) \leq F_{\lambda}(s) \leq$ 
$\left(\lambda s^{p+1} /(p+1)\right)+\left(\lambda s^{q+1} /(q+1)\right), s \in(1, \alpha)$, and, from the fact that $F_{\lambda}(\alpha)=1$, we get that if $s \in(0,1)$, then

$$
\begin{aligned}
1-F_{\lambda}^{2}(s)= & F_{\lambda}^{2}(\alpha)-F_{\lambda}^{2}(s) \\
= & \left(F_{\lambda}(\alpha)-F_{\lambda}(s)\right)\left(F_{\lambda}(\alpha)+F_{\lambda}(s)\right) \\
\geq & \lambda^{2}\left(\frac{1}{p+1}-\frac{s^{p+1}}{p+1}+\frac{\alpha^{q+1}}{q+1}-\frac{1}{q+1}\right) \\
& \times\left(\frac{1}{p+1}+\frac{\alpha^{q+1}}{q+1}+\frac{s^{p+1}}{p+1}-\frac{1}{q+1}\right) \\
\geq & \lambda^{2}\left(\frac{\alpha^{q+1}}{q+1}-\frac{s^{p+1}}{q+1}\right)\left(\frac{\alpha^{q+1}}{q+1}+\frac{s^{p+1}}{q+1}\right) \\
= & \lambda^{2}\left(\frac{\alpha^{2 q+2}}{(q+1)^{2}}-\frac{s^{2 p+2}}{(q+1)^{2}}\right) .
\end{aligned}
$$

And, if $s \in(1, \alpha)$, then

$$
\begin{aligned}
1-F_{\lambda}^{2}(s)= & F_{\lambda}^{2}(\alpha)-F_{\lambda}^{2}(s) \\
= & \left(F_{\lambda}(\alpha)-F_{\lambda}(s)\right)\left(F_{\lambda}(\alpha)+F_{\lambda}(s)\right) \\
\geq & \lambda^{2}\left(\frac{\alpha^{q+1}}{q+1}-\frac{s^{q+1}}{q+1}\right) \\
& \times\left(\frac{1}{p+1}+\frac{\alpha^{q+1}}{q+1}-\frac{1}{q+1}\right. \\
\geq & \quad \lambda^{2}\left(\frac{1}{p+1}+\frac{\alpha^{q+1}}{q+1}-\frac{1}{q+1}\right) \\
= & \lambda^{2} \frac{1}{(q+1)^{2}}\left(\alpha^{2 q+2}-s^{2 q+2}\right) ;
\end{aligned}
$$

therefore,

$$
\begin{aligned}
\alpha \int_{0}^{1} & \frac{F_{\lambda}(\alpha t)}{\sqrt{1-F_{\lambda}^{2}(\alpha t)}} d t \\
\quad & \int_{0}^{\alpha} \frac{F_{\lambda}(s)}{\sqrt{1-F_{\lambda}^{2}(s)}} d s \\
\leq & \int_{0}^{1} \frac{\left(\lambda s^{p+1} /(p+1)\right)+\left(\lambda s^{q+1} /(q+1)\right)}{\sqrt{\lambda^{2}\left(\left(\alpha^{2 q+2} /(q+1)^{2}\right)-\left(s^{2 p+2} /(q+1)^{2}\right)\right)}} d s \\
& +\int_{1}^{\alpha} \frac{\left(\lambda\left(s^{p+1} /(p+1)\right)\right)+\left(\lambda\left(s^{q+1} /(q+1)\right)\right)}{\sqrt{\lambda^{2}\left(\left(\alpha^{2 q+2} /(q+1)^{2}\right)-\left(s^{2 q+2} /(q+1)^{2}\right)\right)}} d s
\end{aligned}
$$

$$
\begin{aligned}
& \leq \int_{0}^{\alpha} \frac{\left(\lambda s^{p+1} /(p+1)\right)+\left(\lambda s^{q+1} /(q+1)\right)}{\sqrt{\lambda^{2}\left(\left(\alpha^{2 q+2} /(q+1)^{2}\right)-\left(s^{2 p+2} /(q+1)^{2}\right)\right)}} d s \\
& =\alpha \int_{0}^{1} \frac{\left((\alpha t)^{p+1} /(p+1)\right)+\left((\alpha t)^{q+1} /(q+1)\right)}{\sqrt{\left(\alpha^{2 q+2} /(q+1)^{2}\right)-\left((\alpha t)^{2 p+2} /(q+1)^{2}\right)}} d t \\
& =\alpha \int_{0}^{1} \frac{\left(\alpha^{p-q} t^{p+1} /(p+1)\right)+\left(t^{q+1} /(p+1)\right)}{\sqrt{\left(1 /(q+1)^{2}\right)-\left(\alpha^{2 p-2 q} t^{2 p+2} /(q+1)^{2}\right)}} d t \\
& \leq \alpha \frac{q+1}{p+1} \int_{0}^{1} \frac{2 t^{p+1}}{\sqrt{1-t^{2 p+2}}} d t .
\end{aligned}
$$

Or

$$
\begin{aligned}
\alpha(\lambda) & \int_{0}^{1} \frac{F_{\lambda}(\alpha t)}{\sqrt{1-F_{\lambda}^{2}(\alpha t)}} d t \\
= & \int_{0}^{\alpha} \frac{F_{\lambda}(s)}{\sqrt{1-F_{\lambda}^{2}(s)}} d s \\
\leq & \int_{0}^{1} \frac{\left(\lambda s^{p+1} /(p+1)\right)+\left(\lambda s^{q+1} /(q+1)\right)}{\sqrt{\lambda^{2}\left(\left(\alpha^{2 q+2} /(q+1)^{2}\right)-\left(s^{2 p+2} /(q+1)^{2}\right)\right)}} d s \\
& +\int_{1}^{\alpha} \frac{\left(\lambda\left(s^{p+1} /(p+1)\right)\right)+\left(\lambda\left(s^{q+1} /(q+1)\right)\right)}{\sqrt{\lambda^{2}\left(\left(\alpha^{2 q+2} /(q+1)^{2}\right)-\left(s^{2 q+2} /(q+1)^{2}\right)\right)}} d s \\
\leq & \int_{0}^{\alpha} \frac{\left(\lambda\left(s^{p+1} /(p+1)\right)\right)+\left(\lambda\left(s^{q+1} /(q+1)\right)\right)}{\sqrt{\lambda^{2}\left(\left(\alpha^{2 q+2} /(q+1)^{2}\right)-\left(s^{2 q+2} /(q+1)^{2}\right)\right)}} d s \\
= & \alpha \int_{0}^{1} \frac{\left(\lambda\left((\alpha t)^{p+1} /(p+1)\right)\right)+\left(\lambda\left((\alpha t)^{q+1} /(q+1)\right)\right)}{\sqrt{\lambda^{2}\left(\left(\alpha^{2 q+2} /(q+1)^{2}\right)-\left((\alpha t)^{2 q+2} /(q+1)^{2}\right)\right)}} d t \\
\leq & \alpha \frac{q+1}{p+1} \int_{0}^{1} \frac{2 t^{p+1}}{\sqrt{1-t^{2 p+2}}} d t .
\end{aligned}
$$

It follows from (26), (29), and (30); we get that $\eta(\lambda)$ is continuous in $(0, \infty)$ and (i) holds. (ii) From (25) and the fact that $1=F_{\lambda}(\alpha(\lambda))$, the following holds.

If $\alpha \leq 1$, then

$$
\lambda \frac{\alpha^{p+1}}{p+1}+\lambda \frac{\alpha^{q+1}}{q+1} \geq 1=F_{\lambda}(\alpha) \geq \lambda \frac{\alpha^{p+1}}{p+1}
$$


thus, if $s \in(0, \alpha)$, then

$$
\begin{aligned}
1-F_{\lambda}^{2}(s)= & F_{\lambda}^{2}(\alpha)-F_{\lambda}^{2}(s) \\
= & \left(F_{\lambda}(\alpha)-F_{\lambda}(s)\right)\left(F_{\lambda}(\alpha)+F_{\lambda}(s)\right) \\
\leq & \lambda^{2}\left(\frac{\alpha^{p+1}}{p+1}-\frac{s^{p+1}}{p+1}+\frac{\alpha^{q+1}}{q+1}-\frac{s^{q+1}}{q+1}\right) \\
& \times\left(\frac{\alpha^{p+1}}{p+1}+\frac{s^{p+1}}{p+1}+\frac{\alpha^{q+1}}{q+1}+\frac{s^{q+1}}{q+1}\right) \\
\leq & 4 \lambda^{2}\left(\frac{\alpha^{2 p+2}}{(p+1)^{2}}-\frac{s^{2 p+2}}{(p+1)^{2}}\right)
\end{aligned}
$$

from (31), we obtain

$$
\begin{aligned}
& \alpha(\lambda) \int_{0}^{1} \frac{F_{\lambda}(\alpha t)}{\sqrt{1-F_{\lambda}^{2}(\alpha t)}} d t \\
& \quad=\int_{0}^{\alpha} \frac{F_{\lambda}(s)}{\sqrt{1-F_{\lambda}^{2}(s)}} d s \\
& \quad \geq \int_{0}^{\alpha} \frac{s^{p+1} /(p+1)}{\sqrt{4\left(\left(\alpha^{2 p+2} /(p+1)^{2}\right)-\left(s^{2 p+2} /(p+1)^{2}\right)\right)}} d s \\
& \quad=\alpha \int_{0}^{1} \frac{(\alpha t)^{p+1} /(p+1)}{\sqrt{4\left(\left(\alpha^{2 p+2} /(p+1)^{2}\right)-\left((\alpha t)^{2 p+2} /(p+1)^{2}\right)\right)}} d t \\
& \quad=\frac{1}{2} \alpha \int_{0}^{1} \frac{t^{p+1}}{\sqrt{1-t^{2 p+2}}} d t .
\end{aligned}
$$

If $\alpha \geq 1$, then $\left(\lambda s^{q+1} /(q+1)\right) \leq\left(\lambda s^{p+1} /(p+1)\right) \leq F_{\lambda}(s) \leq$ $\left(\lambda s^{p+1} /(p+1)\right)+\left(\lambda s^{q+1} /(q+1)\right)$ for $s \in(0,1)$ and $\lambda\left(s^{q+1} /(q+\right.$ $1)) \leq F_{\lambda}(s) \leq\left(\lambda s^{p+1} /(p+1)\right)+\left(\lambda s^{q+1} /(q+1)\right)$ for $s \in(1, \alpha(\lambda))$, and, from the fact that $F_{\lambda}(\alpha(\lambda))=1$, we get the following.

If $s \in(0,1)$, then

$$
\begin{aligned}
1-F_{\lambda}^{2}(s)= & F_{\lambda}^{2}(\alpha(\lambda))-F_{\lambda}^{2}(s) \\
= & \left(F_{\lambda}(\alpha)-F_{\lambda}(s)\right)\left(F_{\lambda}(\alpha(\lambda))+F_{\lambda}(s)\right) \\
\leq & \lambda^{2}\left(\frac{\alpha^{p+1}}{p+1}-\frac{s^{p+1}}{p+1}+\frac{\alpha^{q+1}}{q+1}-\frac{s^{q+1}}{q+1}\right) \\
& \times\left(\frac{\alpha^{p+1}}{p+1}+\frac{\alpha^{q+1}}{q+1}+\frac{s^{p+1}}{p+1}+\frac{s^{q+1}}{q+1}\right) \\
\leq & 6 \lambda^{2}\left(\frac{\alpha^{q+1}}{p+1}-\frac{s^{q+1}}{p+1}\right)\left(\frac{\alpha^{q+1}}{p+1}+\frac{s^{q+1}}{p+1}\right) \\
= & 6 \lambda^{2}\left(\frac{\alpha^{2 q+2}}{(p+1)^{2}}-\frac{s^{2 q+2}}{(p+1)^{2}}\right)
\end{aligned}
$$

and, if $s \in(1, \alpha)$, then

$$
\begin{aligned}
1- & F_{\lambda}^{2}(s) \\
= & F_{\lambda}^{2}(\alpha(\lambda))-F_{\lambda}^{2}(s) \\
= & \left(F_{\lambda}(\alpha)-F_{\lambda}(s)\right)\left(F_{\lambda}(\alpha)+F_{\lambda}(s)\right) \\
\leq & \lambda^{2}\left(\frac{\alpha^{p+1}}{p+1}-\frac{s^{p+1}}{p+1}+\frac{\alpha^{q+1}}{q+1}-\frac{s^{q+1}}{q+1}\right) \\
& \times\left(\frac{\alpha^{p+1}}{p+1}+\frac{\alpha^{q+1}}{q+1}+\frac{s^{p+1}}{p+1}+\frac{s^{q+1}}{q+1}\right) \\
\leq & 4 \lambda^{2}\left(\frac{\alpha^{q+1}}{q+1}-\frac{s^{q+1}}{q+1}\right)\left(\frac{\alpha^{q+1}}{p+1}+\frac{s^{q+1}}{p+1}\right) \\
\leq & 4 \lambda^{2} \frac{1}{(p+1)^{2}}\left(\alpha^{2 q+2}-s^{2 q+2}\right) .
\end{aligned}
$$

Then

$$
\begin{aligned}
& \alpha \int_{0}^{1} \frac{F_{\lambda}(\alpha t)}{\sqrt{1-F_{\lambda}^{2}(\alpha t)}} d t \\
& =\int_{0}^{\alpha} \frac{F_{\lambda}(s)}{\sqrt{1-F_{\lambda}^{2}(s)}} d s \\
& \geq \int_{0}^{1} \frac{\lambda s^{p+1} /(p+1)}{\sqrt{6 \lambda^{2}\left(\left(\alpha^{2 q+2} /(p+1)^{2}\right)-\left(s^{2 q+2} /(p+1)^{2}\right)\right)}} d s \\
& +\int_{1}^{\alpha} \frac{\lambda\left(s^{q+1} /(q+1)\right)}{\sqrt{4 \lambda^{2}\left(\left(\alpha^{2 q+2} /(p+1)^{2}\right)-\left(s^{2 q+2} /(p+1)^{2}\right)\right)}} d s \\
& \geq \int_{0}^{\alpha} \frac{\lambda s^{q+1} /(q+1)}{\sqrt{6 \lambda^{2}\left(\left(\alpha^{2 q+2} /(p+1)^{2}\right)-\left(s^{2 q+2} /(p+1)^{2}\right)\right)}} d s \\
& =\alpha \int_{0}^{1} \frac{\lambda(\alpha t)^{q+1} /(q+1)}{\sqrt{6 \lambda^{2}\left(\left(\alpha^{2 q+2} /(p+1)^{2}\right)-\left((\alpha t)^{2 q+2} /(p+1)^{2}\right)\right)}} d t \\
& =\alpha \int_{0}^{1} \frac{(\alpha t)^{q+1} /(q+1)}{\sqrt{6\left(\left(\alpha^{2 q+2} /(p+1)^{2}\right)-\left((\alpha t)^{2 q+2} /(p+1)^{2}\right)\right)}} d t \\
& =\alpha \frac{p+1}{\sqrt{6}(q+1)} \int_{0}^{1} \frac{t^{q+1}}{\sqrt{1-t^{2 q+2}}} d t .
\end{aligned}
$$

It follows from (26), (29), (33), (36), and Lemma 6 that $\lim _{\lambda \rightarrow 0} \eta(\lambda)=+\infty$ and $\lim _{\lambda \rightarrow \infty} \eta(\lambda)=0$. 
Lemma 9. Let (H1), (H2), and (H4) hold; assume that $0<$ $p \leq q<1$. Then $T(\lambda, \rho)$ has the following properties, for fixed $\lambda>0$ :

(i) $\lim _{\rho \rightarrow 0} T(\lambda, \rho)=0$;

(ii) $\lim _{\rho \rightarrow 0}(\partial / \partial \rho) T(\lambda, \rho)=+\infty$;

(iii) $T(\lambda, \alpha(\lambda))>0$;

(iv) $\left(\partial^{2} / \partial \rho^{2}\right) T(\lambda, \rho)<0$, for $\rho \in(0, \alpha(\lambda))$.

Proof. (i) Since $\rho \rightarrow 0$, we choose $0<\rho<1$; thus from (H2), we get

$$
F_{\lambda}(\rho)-F_{\lambda}(\rho t)=\lambda \int_{\rho t}^{\rho} f(s) d s \geq \rho^{p+1} \frac{\lambda}{p+1}\left(1-t^{p+1}\right)
$$

therefore,

$$
\begin{aligned}
T(\lambda, \rho) & =\rho \int_{0}^{1} \frac{1-\xi}{\sqrt{\xi(2-\xi)}} d t \\
& \leq \rho \int_{0}^{1} \frac{1-\xi}{\sqrt{\rho^{p+1}(\lambda /(p+1))\left(1-t^{p+1}\right)(2-\xi)}} d t \\
& =\rho^{(1-p) / 2} \int_{0}^{1} \frac{1-\xi}{\sqrt{(\lambda /(p+1))\left(1-t^{p+1}\right)(2-\xi)}} d t .
\end{aligned}
$$

Hence, from $p<1$, we have $\lim _{\rho \rightarrow 0} T(\lambda, \rho)=0$.

(ii) From Lemma 5, we have that

$$
\frac{\partial}{\partial \rho} T(\lambda, \rho)=T_{\rho}^{\prime}(\lambda, \rho)=\int_{0}^{1} \frac{\xi(1-\xi)(2-\xi)-\rho \xi^{\prime}}{(\xi(2-\xi))^{3 / 2}} d t
$$

according to the assumption (H2), we have

$$
\begin{aligned}
\xi & =F_{\lambda}(\rho)-F_{\lambda}(\rho t) \\
& =\lambda \int_{\rho t}^{\rho} f(s) d s \\
& \leq \lambda \frac{\rho^{p+1}\left(1-t^{p+1}\right)}{p+1}+\lambda \frac{\rho^{q+1}\left(1-t^{q+1}\right)}{q+1},
\end{aligned}
$$

and, from the assumption (H4), that is, $f(\rho) \geq 3 \rho f^{\prime}(\rho) \geq$ $2 \rho f^{\prime}(\rho), \rho \in(0, \alpha)$, we have $\rho \xi^{\prime} \leq(3 / 2) \xi$; thus

$$
\begin{aligned}
\xi(1-\xi)(2-\xi)-\rho \xi^{\prime} & \geq \xi(1-\xi)(2-\xi)-\frac{3}{2} \xi \\
& =\xi\left((1-\xi)(2-\xi)-\frac{3}{2}\right) .
\end{aligned}
$$

Thus

$$
\begin{aligned}
& \frac{\partial}{\partial \rho} T(\lambda, \rho) \\
& =\int_{0}^{1} \frac{\xi(1-\xi)(2-\xi)-\rho \xi^{\prime}}{(\xi(2-\xi))^{3 / 2}} d t \\
& \geq \int_{0}^{1} \frac{\xi((1-\xi)(2-\xi)-(3 / 2))}{(\xi(2-\xi))^{3 / 2}} d t \\
& \geq \int_{0}^{1}\left(\begin{array}{c}
\left.(1-\xi)(2-\xi)-\left(\frac{3}{2}\right)\right) \\
\quad \times\left(\lambda \rho^{p+1} \frac{\left(1-t^{p+1}\right)}{p+1}+\lambda \frac{\rho^{q+1}\left(1-t^{q+1}\right)}{q+1}\right)^{-1 / 2} \\
\left.\quad \times(2-\xi)^{-3 / 2}\right) d t .
\end{array}\right.
\end{aligned}
$$

Therefore, $\lim _{\rho \rightarrow 0}(\partial / \partial \rho) T(\lambda, \rho)=+\infty$.

(iii) It is proved in the same way as in Lemma 7(ii).

(iv) From Lemma 5, we get that

$$
T_{\rho \rho}^{\prime \prime}(\lambda, \rho)=\frac{3}{\rho} \int_{0}^{1} \frac{(1-\xi)\left(\rho \xi^{\prime}\right)^{2}}{(\xi(2-\xi))^{5 / 2}} d t-\int_{0}^{1} \frac{2 \xi^{\prime}+\rho \xi^{\prime \prime}}{(\xi(2-\xi))^{3 / 2}} d t
$$

From (H1) where $\xi^{\prime \prime} \geq 0$, using the assumption (H4) and the fact that $\xi \leq 1$, we arrive at

$$
\begin{aligned}
3 \rho & (1-\xi)\left(\xi^{\prime}\right)^{2}-\left(2 \xi^{\prime}+\rho \xi^{\prime \prime}\right)(\xi(2-\xi)) \\
& =3 \rho\left(\xi^{\prime}\right)^{2}-3 \rho \xi\left(\xi^{\prime}\right)^{2}-\left(2 \xi^{\prime}+\rho \xi^{\prime \prime}\right)\left(2 \xi-\xi^{2}\right) \\
& =3 \rho\left(\xi^{\prime}\right)^{2}-3 \rho \xi\left(\xi^{\prime}\right)^{2}-4 \xi \xi^{\prime}+2 \xi^{2} \xi^{\prime}-\rho \xi^{\prime \prime}\left(2 \xi-\xi^{2}\right) \\
& <\xi^{\prime}\left(3 \rho \xi^{\prime}-4 \xi\right)(1-\xi)-\rho \xi^{\prime \prime}\left(2 \xi-\xi^{2}\right)<0 .
\end{aligned}
$$

Therefore, $\left(\partial^{2} / \partial \rho^{2}\right) T(\lambda, \rho)<0$, for $\rho \in(0, \alpha)$.

Lemma 10. Assume that $0<p \leq q<1$. Denote $w(\lambda)=$ $\sup \{T(\lambda, \rho) \mid 0<\rho<\alpha(\lambda)\}$. Then

(i) $w(\lambda)$ is strictly decreasing in $(0,+\infty)$;

(ii) $w(\lambda)$ is continuous in $(0,+\infty)$;

(iii) $\lim _{\lambda \rightarrow 0} w(\lambda)=+\infty, \lim _{\lambda \rightarrow+\infty} w(\lambda)=0$.

Proof. (i) From Lemma 9, we get

$$
w(\lambda)=\max \{T(\lambda, \rho) \mid 0<\rho<\alpha(\lambda)\} .
$$

Let $0<\lambda_{1}<\lambda_{2}<+\infty$. From the definition of $\xi=\xi_{\lambda}(\rho, t)$ and Lemma 6 , we obtain

$$
\alpha\left(\lambda_{2}\right)<\alpha\left(\lambda_{1}\right)
$$


And, for $0<\rho \leq \alpha\left(\lambda_{2}\right)$ and $0<t<1$,

$$
\xi_{\lambda_{1}}(\rho, t)=\lambda_{1} \int_{\rho t}^{\rho} f(s) d s<\xi_{\lambda_{2}}(\rho, t)=\lambda_{2} \int_{\rho t}^{\rho} f(s) d s
$$

It follows that, for $0<\rho \leq \alpha\left(\lambda_{2}\right)$,

$$
\begin{aligned}
& \rho \int_{0}^{1} \frac{1-\xi_{\lambda_{2}}(\rho, t)}{\sqrt{\xi_{\lambda_{2}}(\rho, t)\left(2-\xi_{\lambda_{2}}(\rho, t)\right)}} d t \\
& \quad<\rho \int_{0}^{1} \frac{1-\xi_{\lambda_{1}}(\rho, t)}{\sqrt{\xi_{\lambda_{1}}(\rho, t)\left(2-\xi_{\lambda_{1}}(\rho, t)\right)}} d t
\end{aligned}
$$

thus,

$$
T\left(\lambda_{2}, \rho\right)<T\left(\lambda_{1}, \rho\right)
$$

Hence, $w\left(\lambda_{2}\right)<w\left(\lambda_{1}\right)$, which implies that $w(\lambda)$ is strictly decreasing in $(0,+\infty)$.

(ii) Let $\lambda_{0} \in(0,+\infty)$. Since $F_{\lambda_{0}}\left(\alpha\left(\lambda_{0}\right)\right)=1$, there exists $\delta>0$ such that, for $0<\lambda \leq \lambda_{0}+\delta$ and $0<\rho \leq \alpha\left(\lambda_{0}\right)+\delta$,

$$
F_{\lambda}(\rho)=\lambda \int_{0}^{\rho} f(s) d s<\left(\lambda_{0}+\delta\right) \int_{0}^{\alpha\left(\lambda_{0}\right)+\delta} f(s) d s<\frac{3}{2}
$$

It follows that $\xi=\xi_{\lambda}(\rho, t)<3 / 2$, for $0<\rho \leq \alpha\left(\lambda_{0}\right)+\delta$, $t \in[0,1]$. Further,

$$
\begin{gathered}
\xi_{\lambda}(\rho, t)=\lambda \int_{\rho t}^{\rho} f(s) d s \geq \lambda \frac{\rho^{p+1}\left(1-t^{p+1}\right)}{p+1}, \quad \rho \leq 1, \\
\xi_{\lambda}(\rho, t)=\lambda \int_{\rho t}^{\rho} f(s) d s \geq \lambda \frac{\rho^{q+1}\left(1-t^{q+1}\right)}{q+1}, \\
\rho>1, \quad \rho t \geq 1, \\
\xi_{\lambda}(\rho, t)=\lambda \int_{\rho t}^{\rho} f(s) d s \geq \lambda \frac{\rho^{q+1}\left(1-t^{p+1}\right)}{q+1}, \\
\rho>1, \quad \rho t \leq 1 .
\end{gathered}
$$

Define

$$
\begin{aligned}
& \psi_{1}(\lambda, \rho)=\int_{0}^{1} \frac{1-\xi_{\lambda}(\rho, t)}{\sqrt{\int_{\rho^{-p} t}^{\rho^{-p}} f\left(\rho^{p+1} s\right) d s\left(2-\xi_{\lambda}(\rho, t)\right)}} d t, \quad \rho \leq 1, \\
& \psi_{2}(\lambda, \rho)=\int_{0}^{1} \frac{1-\xi_{\lambda}(\rho, t)}{\sqrt{\int_{\rho^{-q} t}^{\rho^{-q}} f\left(\rho^{q+1} s\right) d s\left(2-\xi_{\lambda}(\rho, t)\right)}} d t,
\end{aligned}
$$

Thus, if $\rho \leq 1$, then

$$
\begin{aligned}
\psi_{1} & (\lambda, \rho) \\
& =\int_{0}^{1} \frac{1-\xi_{\lambda}(\rho, t)}{\sqrt{\int_{\rho^{-p} t}^{\rho^{-p}} f\left(\rho^{p+1} s\right) d s\left(2-\xi_{\lambda}(\rho, t)\right)}} d t \\
& \leq \int_{0}^{1} \frac{1-\xi_{\lambda}(\rho, t)}{\sqrt{\int_{\rho^{-p} t}^{\rho^{-p}} \rho^{p(p+1)} s^{p} d s\left(2-\xi_{\lambda}(\rho, t)\right)}} d t \\
& \leq \sqrt{2} \int_{0}^{1} \frac{1-\xi_{\lambda}(\rho, t)}{\sqrt{\int_{\rho^{-p} t}^{\rho^{-p}} \rho^{p(p+1)} s^{p} d s}} d t \\
& \leq \sqrt{2} \sqrt{p+1} \int_{0}^{1} \frac{1-\xi_{\lambda}(\rho, t)}{\sqrt{1-t^{p+1}}} d t \\
& \leq \sqrt{2} \sqrt{p+1} \int_{0}^{1} \frac{1}{\sqrt{1-t^{p+1}}} d t
\end{aligned}
$$

and, if $\rho>1$, then

$$
\begin{aligned}
& \psi_{2}(\lambda, \rho) \\
& =\int_{0}^{1} \frac{1-\xi_{\lambda}(\rho, t)}{\sqrt{\int_{\rho^{-q} t}^{\rho^{-q}} f\left(\rho^{q+1} s\right) d s\left(2-\xi_{\lambda}(\rho, t)\right)}} d t \\
& \leq \int_{0}^{1} \frac{1-\xi_{\lambda}(\rho, t)}{\sqrt{\int_{\rho^{-q} t}^{\rho^{-q}} \rho^{q(q+1)} s^{q} d s\left(2-\xi_{\lambda}(\rho, t)\right)}} d t \\
& \leq \sqrt{2} \int_{0}^{1} \frac{1-\xi_{\lambda}(\rho, t)}{\sqrt{\int_{\rho^{-q}}^{\rho^{-q}} \rho^{q(q+1)} s^{q} d s}} d t \\
& \leq \sqrt{2} \sqrt{q+1} \int_{0}^{1} \frac{1-\xi_{\lambda}(\rho, t)}{\sqrt{1-t^{p+1}}} d t \\
& \leq \sqrt{2} \sqrt{q+1} \int_{0}^{1} \frac{1}{\sqrt{1-t^{p+1}}} d t .
\end{aligned}
$$

It follows that the singular integral in (53) and (54) converges uniformly in $\left[0, \lambda_{0}+\delta\right] \times\left[0, \alpha\left(\lambda_{0}\right)+\delta\right]$, and hence $\psi_{1}(\lambda, \rho)$ and $\psi_{2}(\lambda, \rho)$ are continuous in $\left[0, \lambda_{0}+\delta\right] \times\left[0, \alpha\left(\lambda_{0}\right)+\delta\right]$. By (i), we have

$$
\lim _{\lambda \rightarrow \lambda_{0}^{-}} w(\lambda) \geq w\left(\lambda_{0}\right) \geq \lim _{\lambda \rightarrow \lambda_{0}^{+}} w(\lambda)
$$

We will see that the equalities hold in (55). If this does not hold, then we may assume, for instance,

$$
\lim _{\lambda \rightarrow \lambda_{0}^{-}} w(\lambda)>w\left(\lambda_{0}\right)
$$

Choose an increasing sequence $\left\{\lambda_{n}\right\} \subset\left(0, \lambda_{0}\right)$ satisfying $\lim _{n \rightarrow \infty} \lambda_{n}=\lambda_{0}$ and then choose $\rho_{n} \in\left(0, \alpha\left(\lambda_{n}\right)\right)$ such that

$$
\alpha\left(\lambda_{n}\right)<\alpha\left(\lambda_{0}\right)+\delta, \quad w\left(\lambda_{n}\right)=T\left(\lambda_{n}, \rho_{n}\right) .
$$


Since $\lim _{n \rightarrow \infty} \alpha\left(\lambda_{n}\right)=\alpha\left(\lambda_{0}\right)$, we may assume that there exists $\rho_{0} \in\left[0, \alpha\left(\lambda_{0}\right)\right]$ such that $\lim _{n \rightarrow \infty} \rho_{n}=\rho_{0}$; by the continuity of $\psi_{1}(\lambda, \rho)$ and $\psi_{2}(\lambda, \rho)$, we have

$$
\begin{aligned}
\lim _{n \rightarrow \infty} w\left(\lambda_{n}\right) & =\lim _{n \rightarrow \infty} T\left(\lambda_{n}, \rho_{n}\right) \\
& =\lim _{n \rightarrow \infty} \sqrt{\frac{\rho_{n}^{1-p}}{\lambda_{n}}} \psi_{1}\left(\lambda_{n}, \rho_{n}\right) \\
& =\sqrt{\frac{\rho_{0}^{1-p}}{\lambda_{0}}} \psi_{1}\left(\lambda_{0}, \rho_{0}\right) \\
& =T\left(\lambda_{0}, \rho_{0}\right) \leq w\left(\lambda_{0}\right), \quad \rho_{0} \leq 1,
\end{aligned}
$$

or

$$
\begin{aligned}
& \lim _{n \rightarrow \infty} w\left(\lambda_{n}\right)=\lim _{n \rightarrow \infty} T\left(\lambda_{n}, \rho_{n}\right) \\
&=\lim _{n \rightarrow \infty} \sqrt{\frac{\rho_{n}^{1-q}}{\lambda_{n}}} \psi_{2}\left(\lambda_{n}, \rho_{n}\right) \\
&=\sqrt{\frac{\rho_{0}^{1-q}}{\lambda_{0}}} \psi_{2}\left(\lambda_{0}, \rho_{0}\right)=T\left(\lambda_{0}, \rho_{0}\right) \leq w\left(\lambda_{0}\right), \\
& \quad \rho_{0}>1,
\end{aligned}
$$

which contradicts (56). Therefore, $\lim _{\lambda \rightarrow \lambda_{0}^{-}} w(\lambda)=w\left(\lambda_{0}\right)$. Similarly, we have $\lim _{\lambda \rightarrow \lambda_{0}^{+}} w(\lambda)=w\left(\lambda_{0}\right)$. So $w(\lambda)$ is continuous at $\lambda_{0}$.

(iii) Note that $\lim _{\lambda \rightarrow+\infty} \alpha(\lambda)=0$, for any $\lambda>0,0<\rho \leq$ $\alpha(\lambda)$, and $0<t<1$, and, from (55), we have

$$
\begin{aligned}
& \lim _{\lambda \rightarrow+\infty} w(\lambda) \\
& =\lim _{\lambda \rightarrow+\infty} \max _{0<\rho \leq \alpha(\lambda)} T(\lambda, \rho) \\
& \leq \lim _{\lambda \rightarrow+\infty} \sqrt{2} \rho \\
& \quad \times \int_{0}^{1}(1 \\
& \times\left(\lambda \operatorname { m a x } \left\{\frac{\rho^{q+1}\left(1-t^{q+1}\right)}{q+1},\right.\right. \\
& \left.\left.\left.\leq \lim _{\lambda \rightarrow+\infty} \sqrt{2} \rho \int_{0}^{1} \frac{\rho^{p+1}\left(1-t^{p+1}\right)}{p+1}\right\}\right)^{-1 / 2}\right) d t \\
& \sqrt{\lambda\left(\rho^{2}\left(1-t^{q+1}\right) /(q+1)\right)}
\end{aligned}
$$

$$
\begin{aligned}
& \leq \lim _{\lambda \rightarrow+\infty} \sqrt{2}(q+1) \int_{0}^{1} \frac{1}{\sqrt{\lambda\left(1-t^{p+1}\right)}} d t \\
& =0 .
\end{aligned}
$$

Hence, $\lim _{\lambda \rightarrow+\infty} w(\lambda)=0$.

Fix $\lambda_{1}>0$ and $0<\rho_{1}<\alpha\left(\lambda_{1}\right)$. For $\lambda<\lambda_{1}$, the definition of $w(\lambda)$ and the fact that $\alpha\left(\lambda_{1}\right)<\alpha(\lambda)$ imply $w(\lambda) \geq T\left(\lambda, \rho_{1}\right)$. Since $\lim _{\lambda \rightarrow 0} \xi_{\lambda}\left(\rho_{1}, t\right)=0$,

$$
\begin{array}{r}
\lim _{\lambda \rightarrow 0} T\left(\lambda, \rho_{1}\right) \\
=\lim _{\lambda \rightarrow 0} \rho_{1} \int_{0}^{1} \frac{1-\xi_{\lambda}\left(\rho_{1}, t\right)}{\sqrt{\xi_{\lambda}\left(\rho_{1}, t\right)\left(2-\xi_{\lambda}\left(\rho_{1}, t\right)\right)}} d t \\
\geq \lim _{\lambda \rightarrow 0} \rho_{1} \int_{0}^{1}\left(1-\xi_{\lambda}\left(\rho_{1}, t\right)\right) \\
\times\left(\lambda \left\{\frac{\rho_{1}^{q+1}\left(1-t^{q+1}\right)}{q+1}\right.\right. \\
\left.+\frac{\rho_{1}^{p+1}\left(1-t^{p+1}\right)}{p+1}\right\}
\end{array}
$$

$$
\left.\times\left(2-\xi_{\lambda}\left(\rho_{1}, t\right)\right)\right)^{-1 / 2} d t
$$

$=+\infty$.

Hence, $\lim _{\lambda \rightarrow 0} w(\lambda)=+\infty$.

\section{The Proofs of the Main Results}

Proof of Theorem 1. Similar to the proof of Theorem 1.1 [3], it follows from Lemmas 7 and 8 that the results are easy to prove.

Proof of Theorem 2. Similar to the proof of Theorem 1.2 [3], it follows from Lemmas 8,9 , and 10 that the results are easy to prove.

\section{Conflict of Interests}

The authors declare that there is no conflict of interests regarding the publication of this paper.

\section{Acknowledgments}

This paper was supported by the NSFC (no. 11361054), the SRFDP (no. 20126203110004), and the Gansu Provincial National Science Foundation of China (no. 1208RJZA258). 


\section{References}

[1] P. Habets and P. Omari, "Multiple positive solutions of a onedimensional prescribed mean curvature problem," Communications in Contemporary Mathematics, vol. 9, no. 5, pp. 701-730, 2007.

[2] P. Habets and P. Omari, "Positive solutions of an indefinite prescribed mean curvature problem on a general domain," Advanced Nonlinear Studies, vol. 4, no. 1, pp. 1-13, 2004.

[3] W. Li and Z. Liu, "Exact number of solutions of a prescribed mean curvature equation," Journal of Mathematical Analysis and Applications, vol. 367, no. 2, pp. 486-498, 2010.

[4] H. Pan, "One-dimensional prescribed mean curvature equation with exponential nonlinearity," Nonlinear Analysis. Theory, Methods and Applications, vol. 70, no. 2, pp. 999-1010, 2009.

[5] F. Obersnel and P. Omari, "Existence and multiplicity results for the prescribed mean curvature equation via lower and upper solutions," Differential Integral Equations, vol. 22, pp. 853-880, 2009.

[6] F. Obersnel and P. Omari, "Positive solutions of the Dirichlet problem for the prescribed mean curvature equation," Journal of Differential Equations, vol. 249, no. 7, pp. 1674-1725, 2010.

[7] X. Zhang and M. Feng, "Exact number of solutions of a onedimensional prescribed mean curvature equation with concaveconvex nonlinearities," Journal of Mathematical Analysis and Applications, vol. 395, pp. 493-402, 2012.

[8] P. Korman and Y. Li, "Global solution curves for a class of quasilinear boundary-value problems," Proceedings of the Royal Society of Edinburgh A: Mathematics, vol. 140, no. 6, pp. 11971215, 2010.

[9] P. Korman, "Uniqueness and exact multiplicity of solutions for a class of Dirichlet problems," Journal of Differential Equations, vol. 244, no. 10, pp. 2602-2613, 2008.

[10] P. Benevieri, J. M. DoÓ, and E. S. de Medeiros, "Periodic solutions for nonlinear systems with mean curvature-like operators," Nonlinear Analysis. Theory, Methods and Applications, vol. 65, no. 7, pp. 1462-1475, 2006.

[11] D. Bonheure, P. Habets, F. Obersnel, and P. Omari, "Classical and non-classical solutions of a prescribed curvature equation," Journal of Differential Equations, vol. 243, no. 2, pp. 208-237, 2007.

[12] V. K. Le, "Variational method based on finite dimensional approximation in a generalized prescribed mean curvature problem," Journal of Differential Equations, vol. 246, no. 9, pp. 3559-3578, 2009.

[13] A. Ambrosetti, H. Brezis, and G. Cerami, "Combined effects of concave and convex nonlinearities in some elliptic problems," Journal of Functional Analysis, vol. 122, no. 2, pp. 519-543, 1994.

[14] M. Tang, "Exact multiplicity for semilinear elliptic Dirichlet problems involving concave and convex nonlinearities," Proceedings of the Royal Society of Edinburgh A, vol. 133, no. 3, pp. 705-717, 2003.

[15] Z. Liu, "Exact number of solutions of a class of two-point boundary value problems involving concave and convex nonlinearities," Nonlinear Analysis. Theory, Methods and Applications, vol. 46, no. 2, pp. 181-197, 2001.

[16] S. H. Wang and T. S. Yeh, "On the exact structure of positive solutions of an Ambrosetti-Brezis-Cerami problem and its generalization in one space variable," Differential Integral Equations, vol. 17, pp. 17-44, 2004. 


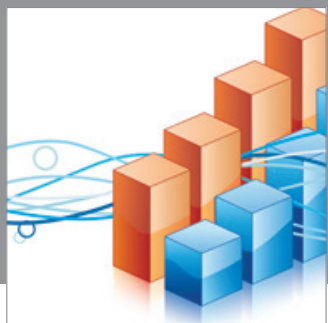

Advances in

Operations Research

mansans

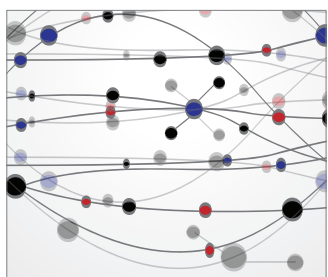

The Scientific World Journal
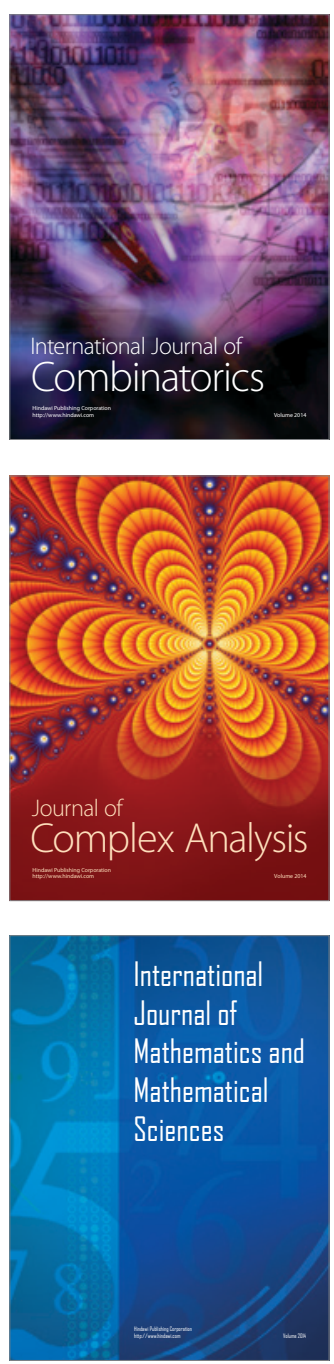
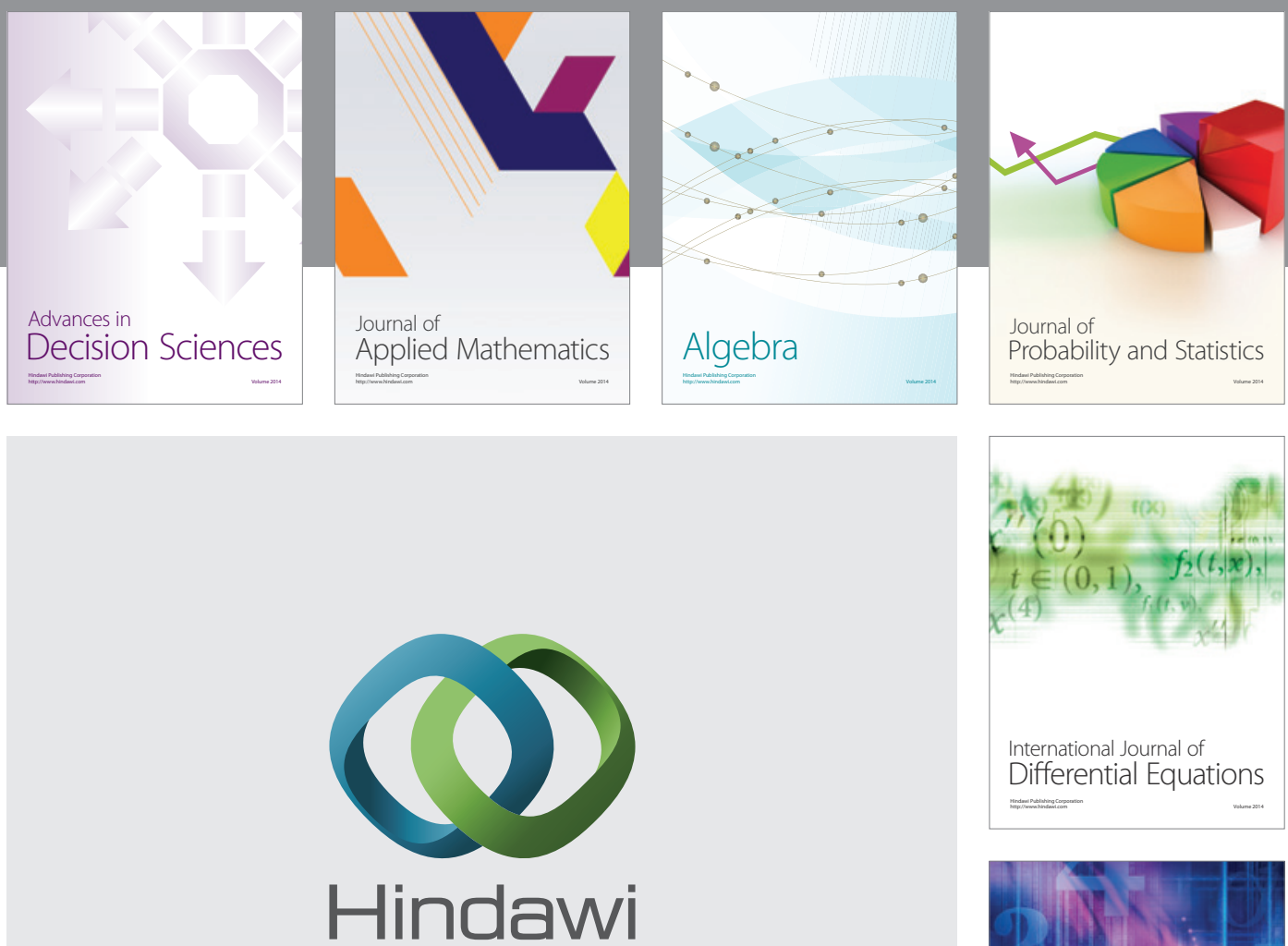

Submit your manuscripts at http://www.hindawi.com
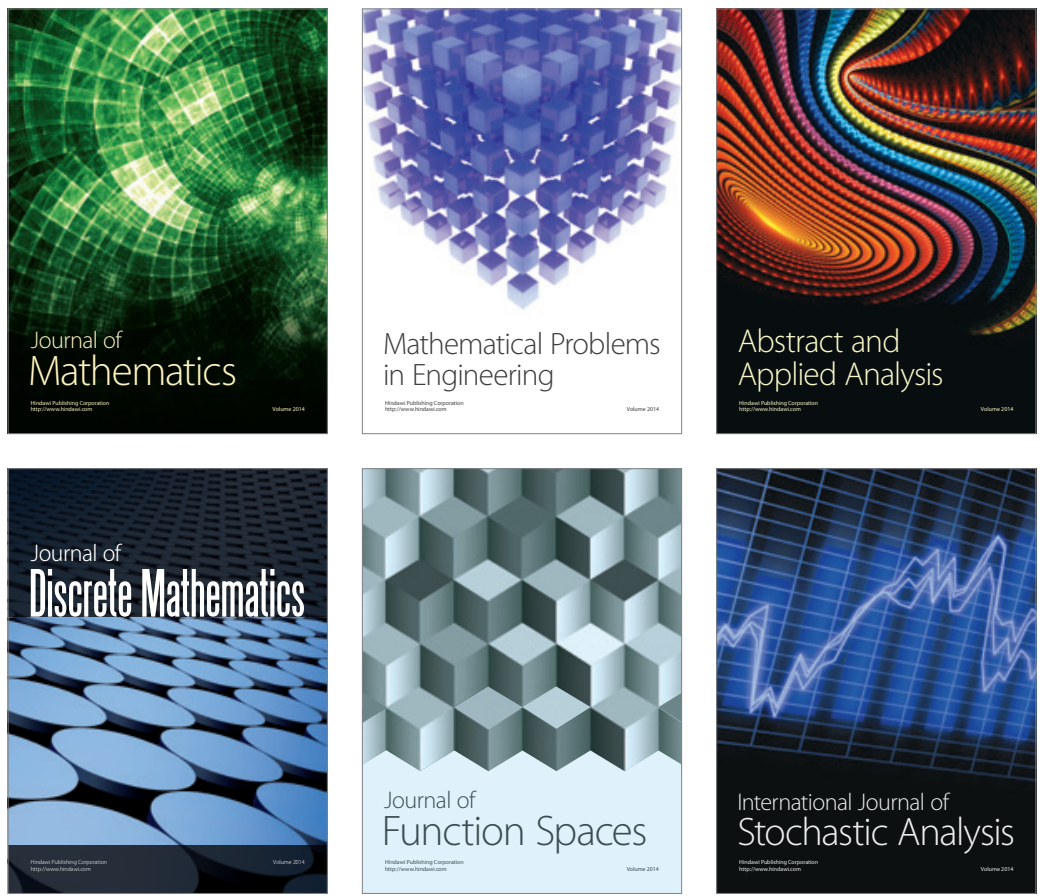

Journal of

Function Spaces

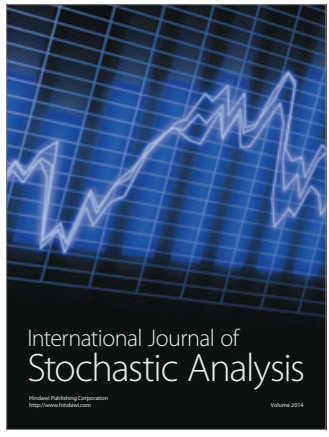

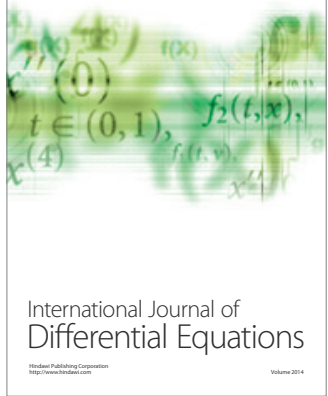
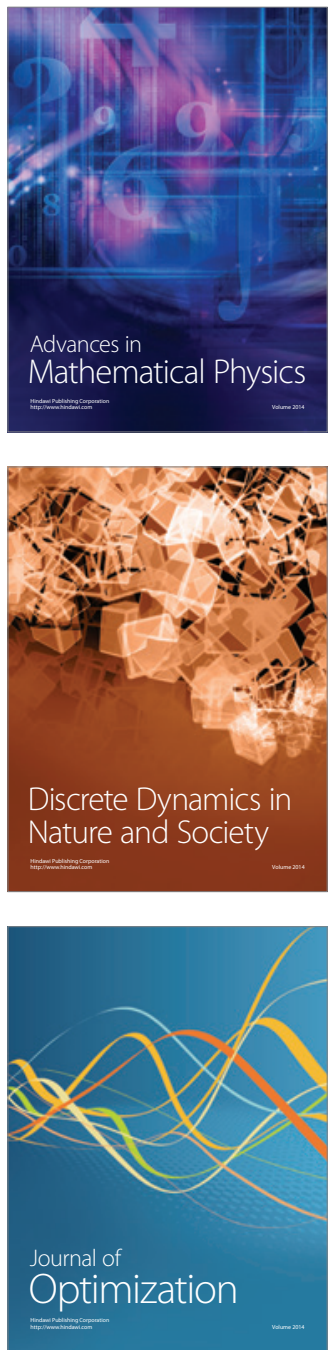